\section{Geopolíticas del cotidiano en la frontera patagónica: Las dinámicas del habitar en torno a los Campos de Hielo Patagónicos}

\section{Everyday life geopolitics at the Patagonian border: The dynamics of dwelling around the Patagonian Ice Fields}

\author{
Sofia Pérez ${ }^{* *}$
}

\section{Resumen}

El siguiente artículo propone analizar la emergencia de una geopolítica desde las dinámicas cotidianas de los habitantes, en una zona donde persiste una tensión diplomática histórica, como es el caso de la frontera chileno-argentina sobre los Campos de Hielo Sur en la Patagonia. Desde la mirada de la geografía crítica e inspirándose de la corriente feminista de la geopolítica, se lleva a cabo una investigación donde se plantea la necesidad de repensar la frontera más allá del marco de las políticas que apelan al reforzamiento del EstadoNación, a la seguridad y al control del territorio. Se explorarán las narrativas geopolíticas que emergen desde el habitar, las prácticas sociales que espacializan la frontera, como también su materialización política. Se consideran para este estudio las ciudades de Villa O'Higgins en Chile y la de El Chaltén en Argentina, en tanto que comunidades ancladas en el margen de los territorios nacionales, y donde el turismo se convierte en un agente que redefine las practicas espaciales en zona fronteriza.

Palabras clave: Geopolítica del cotidiano, frontera, habitar, Campos de Hielo, Patagonia.

\begin{abstract}
The following article proposes to analyse the emergency of geopolitics from the everyday life dynamics of its inhabitants, in an area where diplomatic historical tensions persist, as is the case in the Southern Icefields of Patagonia on the Chilean-Argentinian border. The investigation will be based on a critical geography approach and inspired on feminist geopolitics, where it
\end{abstract}


is argued that there is a need to represent the border beyond the political frame that summons the reinforcement of the Nation-State, security, and the control of territory. The article explores the geopolitical narratives that emerge from dwelling, the social practices that spatialize the border, as their political materialization. For this study, the towns of Villa O'Higgins in Chile and El Chaltén in Argentina, will be considered as communities rooted at the edge of the national territories, where tourism redefines the spatial practices on the border zone.

Key words: Everyday life geopolitics, border, dwelling, Ice Fields, Patagonia.

\section{Introducción}

\subsection{La complejidad del espacio fronterizo patagónico entre Chile y Argentina.}

Las fronteras en América Latina siguen siendo un tema sensible entre algunos países, donde las identidades culturales y dinámicas de circulación eran anteriores a la imposición de un límite entre dos territorios nacionales. Desde una mirada geopolítica, la frontera en el sur del continente está impregnada de una larga historia de conflictos, guerras y arbitrajes para su definición durante el proceso de la formación de los nuevos Estados-Nacionales (Amilhat-Szary 2005; Foucher 1991). En el caso de Chile y Argentina, si bien la demarcación del límite internacional a través de hitos ha sido un proceso centenario de negociaciones, la estabilidad de las relaciones internacionales entre ambos países ha permitido avanzar hacia a una integración pensada como una estrategia para el desarrollo económico de la región (Lacoste 1999). Esta etapa de integración bi-nacional dinamiza la frontera en cuanto a su estructura social, espacial, económica y política. Plataformas internacionales como el "Mercado Común del Sur" (MERCOSUR), la "Asociación Latinoamericana de Integración" (ALADI), la "Unión de Naciones Suramericanas" (UNASUR), y la "Iniciativa para la Integración de la Infraestructura Regional Suramericana" (IIRSA), han permitido avanzar hasta lograr consolidar lazos colaborativos en materia de política internacional. Sin embargo, se continúa manteniendo cierta 'obsesión' por las fronteras entre ambos países, algo que se relaciona con lo que el geógrafo y diplomático francés Michel Foucher define como "banalización de las prácticas de seguridad" (Foucher 2018:10). Es decir, esta obsesión se refleja en la necesidad constante de densificar hitos demarcatorios y a los controles aduaneros, los cuales en este caso, conciernen no solo a la circulación de personas, sino que también a los alimentos a través del Servicio Agrícola Ganadero en Chile (SAG), y del Servicio Nacional de Calidad y Seguridad Agroalimentaria (SENASA) en Argentina.

De forma paralela a esta cuota de obsesión por la seguridad interna, ambos países hacen un esfuerzo conjunto en la generación de herramientas y políticas que beneficien la integración y el desarrollo de sus territorios siguiendo una agenda bilateral. Esta se traduce en una plataforma más íntima que renueva los antiguos Comités de Frontera definidos luego de la firma de tratado de Paz y Amistad (1984) y transformándolos en Comités de Integración. Estos fueron definidos en el Tratado de Maipú de Integración y Cooperación en el año 2009 como comités binacionales destinados a constituirse en tanto que:

“(...) foros de encuentro y colaboración entre los sectores público y privado de las provincias argentinas y regiones chilenas para promover la integración en el ámbito 
subnacional, con el apoyo de los organismos nacionales, provinciales, regionales y municipales"'.

En el caso de la Patagonia Austral, el comité sesiona anualmente en tanto que Comité de Integración Austral Sur-Sur. Estos foros binacionales integran diferentes escalas de poder institucional de cada país, y refuerzan la estabilidad política buscando soluciones conjuntas a problemáticas sociales, económicas y de infraestructura a ambos lados de la frontera, desarrollando los territorios en torno a ella, es decir, generando regiones de frontera. Es así como la línea que dividía el mapa en una dimensión cartesiana adquiere una dimensión espacial, pasando a convertirse en una 'región fronteriza' (borderlands), dinamizada a partir de las circulaciones y los flujos comerciales entre ellas (Amilhat-Szary 2015). Estas regiones son muy difíciles de determinar en su extensión considerando la escala local, pues el efecto de la existencia de una frontera genera complejidad. Una frontera tiene el poder de transformar los territorios, alcanzando distintas escalas como la experiencia del cotidiano, paisajes y políticas públicas (Amilhat-Szary 2015, Núñez et al 2017b).

En la actualidad, el concepto de región fronteriza considerando un área a ambos lados del límite internacional, no ha sido realmente integrado en los instrumentos de planificación en materia de políticas públicas chilenas o argentinas. En el caso de las Comunidad Andina (1969) se ha acordado la creación de Zonas de Integración Fronterizas (ZIF), las cuales han sido concebidas principalmente entre los países miembros (Bolivia, Colombia, Ecuador y Perú),

Tratado de Maipú de Integración y Cooperación entre la República de Chile y la República de Argentina. Art. 15. 30 de octubre de 2009. y no en el de los países asociados como son Argentina y Chile. En estos últimos, se continúa reproduciendo la noción de "zona fronteriza" en el caso de Chile, y el de "zona de frontera" en el caso de Argentina, desde una perspectiva nacional y territorializada.

En Argentina la zona de seguridad de frontera fue definida en $1944^{2}$, considerando una franja de terreno medida a partir del límite internacional hasta unos 150 kilómetros de extensión hacia el interior, como una herramienta de defensa nacional. Estas zonas de seguridad se reglamentaban en base a una serie de restricciones respecto a la propiedad y al uso del suelo en la zona de frontera. Sin embargo, durante los años '70s una nueva visión de los territorios limítrofes surge frente a la necesidad de poblar e integrar los espacios fronterizos proponiendo la "promoción para el desarrollo de las zonas de frontera"3. En ella se consideraban áreas de frontera donde las prioridades se enfocaban a dar un apoyo financiero, infraestructura, facilidades de acceso a la tierra, y todo lo necesario con el fin de estimular la "radicación y arraigo de la población"4. Luego estas figuras territoriales fueron definidas en $1987^{5}$ con una distinción jurídica entre 'zona de seguridad de frontera' y 'zona de frontera para el desarrollo'. Posteriormente fueron reunificadas bajo un régimen de zona de áreas de frontera en $1994^{6}$ al ser consideradas como concurrentes, reconfigurando esta superposición de zonas

Decreto de ley N. 15.385. Buenos Aires, 13 de junio de 1944.

Ley 18.575, Buenos Aires, 30 de enero de1970.

Ibid., Articulo 6.

Decreto Nacional 1182/1987/ Buenos Aires, 23 de julio de 1987.

Decreto Nacional 887/1994, Buenos Aires, 6 de junio de 1994. 
para cada provincia, y excluyendo a los tejidos urbanos de las restricciones de seguridad.

En el caso de Chile, la zona fronteriza no es regulada de la misma manera, siendo definida y gestionada por la Dirección Nacional de Fronteras y Límites del Estado (DIFROL), quienes deben promover políticas para:

“(..) el desarrollo y progreso de las zonas fronterizas, entendiéndose bajo esta designación a las partes del territorio nacional que se determinen por S. E. el Presidente de la República como áreas de apoyo de la línea limítrofe internacional”’

Las dimensiones de la zona fronteriza, las formas de promover su desarrollo, y la relación con los intereses de seguridad y defensa, son criterios reservados de dicho organismo para la determinación de las zonas fronterizas ${ }^{8}$. Por ejemplo, la totalidad la región de Aysén es considerada hoy en día como zona fronteriza, siendo que en otras regiones de Chile las zonas fronterizas abarcanáreas mucho más pequeñas ${ }^{9}$. Las políticas de seguridad en ella se reducen a restricciones sobre la adquisición de tierras e inmuebles por parte de extranjeros en zonas fronterizas, definiendo una franja de seguridad de $10 \mathrm{~km}$ a partir del límite internacional ${ }^{10}$.

Respecto a las zonas fronterizas sensibles de la Patagonia y la labor de la Comisión Mixta de Límites, ambos países solicitaron un

Art. 20, Decreto 559. Reglamento orgánico de la Dirección de Fronteras y Limites del Estado. 1967, Santiago, Chile.

8 Op. Cit. Art. 21. "La determinación de las zonas fronterizas estará sujeta en el espacio de influencia que sea necesario, a las condiciones que presenten, integradamente todos los factores que concurren a la estructuración de la territorialidad".

9 Ver www.difrol.cl

10 Ver Decreto Ley 1939, Santiago 1977 último arbitraje internacional en $1991^{11}$ para resolver los 24 puntos fronterizos pendientes de demarcación (Martinic 2005). Entre ellos se encontraba la zona de los Campos de Hielo Sur $^{12}$ (en adelante (CHS), más puntualmente, la Laguna del Desierto y el monte Fitz Roy. El asunto fue resuelto en parte con una sentencia arbitral en 1994 la cual favoreció la propuesta de limite presentada por la comisión argentina (García 1996; ONU 2006 ${ }^{13}$ ). Si bien se definió el recorrido del límite internacional entre el Monte Fitz Roy y el cerro Daudet en el acuerdo firmado en 1998 por ambos países, la demarcación del tramo $\mathrm{B}$ de dicho límite continúa pendiente ${ }^{14}$.

Las tensiones generadas a partir del enfrentamiento que tuvo lugar en las cercanías de la Laguna del Desierto en 1965, y su posterior arbitraje en 1994, despertaron el interés de los especialistas locales en generar discursos geopolíticos. Al respecto existe una larga lista de publicaciones la cual afloró durante los años '60s y '90s en ambos países, conectando círculos militares, policiales, y académicos. Sin embargo, se mantuvo una perspectiva más bien clásica de la geopolítica, marcada por tendencias nacionalistas, enfocada en la seguridad y la defensa del territorio.

En cuanto a la frontera que divide los $\mathrm{CHS}$ en los alrededores del Monte Fitz Roy, ésta ha

Declaración conjunta presidencial Argentina-Chile. Buenos Aires, 1991.

12 También llamados en Argentina "Hielos Continentales Patagónicos" (HCP).

13 ONU. 2006. Reports of International Arbitral Awards. Boundary dispute between Argentina and Chile concerning the frontier line between boundary post 62 and Mount Fitzroy. Vol. XXII, 21 oct. 1994, pp. 3-149.

14 Acuerdo firmado en 1998 en Buenos Aires por los presidentes Menem y Aylwin, y ratificado por el Decreto N. 1.155 de 1999 en Santiago. 
cambiado su antiguo estatus de zona de frontera de seguridad o sensible para transformarse hoy en día en un área de integración.

Imagen 1. Área de estudio aproximada del habitar fronterizo.

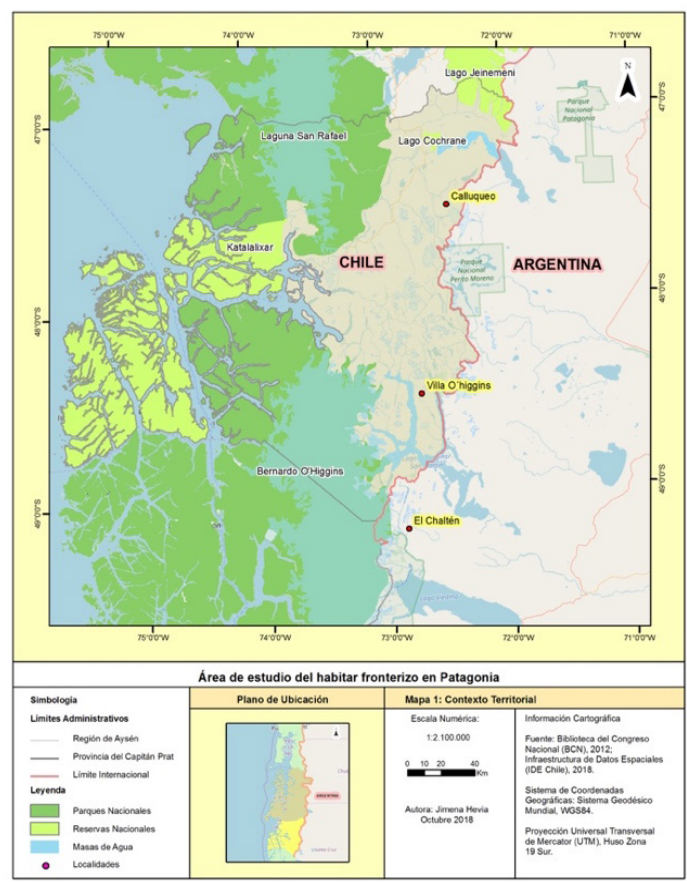

Fuente: Cartografía realizada por la autora.

En esta zona la circulación transfronteriza de personas ha ido en aumento tras el alza del turismo en el Parque Nacional Los Glaciares (Argentina). No obstante, el desarrollo de los territorios adyacentes de manera integrada se ha visto supeditada a una temporalidad bastante más lenta en materia de toma de decisiones en las instancias de las reuniones binacionales del Comité de Integración Austral. Los habitantes ven su cotidianidad directamente afectada por los avances o estancamientos de los proyectos, por sobre todo en lo que respecta a la conectividad trasandina en áreas de difícil acceso terrestre. Se vuelve necesario entonces cambiar de escala en un análisis geopolítico crítico, siguiendo la corriente de la geografía feminista, es decir, una escala que permita visibilizar los problemas de las comunidades que han sido postergadas en el margen de ambos países. Examinar la geopolítica del cotidiano (everyday geopolitics) en las formas de habitar el espacio fronterizo de la Patagonia, permite revelar la eficacia o ineficacia de las relaciones bi-nacionales diplomáticas en el marco de la integración a partir de las experiencias y prácticas cotidianas locales. Siguiendo esta línea, el objetivo de este artículo es replantear los cuestionamientos en torno a los estudios de frontera, incluyendo la perspectiva de la geopolítica crítica y el cambio de paradigma espacial planteado por la corriente feminista de la geopolítica respecto a la escala de análisis. De esta manera el espacio fronterizo se corporaliza permitiendo visibilizar a las personas, en este caso a los habitantes, y las narrativas geopolíticas que se construyen y emergen desde su cotidiano como efecto de las políticas nacionales (Hyndman, 2001). Esta contracorriente geopolítica que emerge desde lo local puede producir también "micromecanismos de poder" (Foucault 1980:101 Cit. en Secor 2001:193), los cuales tendrán una influencia en un contexto de políticas más globales, conformando entonces una dinámica de influencias de poderes top-down y bottomup que se sobreponen y constituyen a su vez el espacio político (Secor 2001). En consecuencia, el interés de este análisis no son los márgenes estáticos de los Estados nacionales, ni poner en discusión el trazado del límite internacional, sino que poner en evidencia las dinámicas del habitar en una región fronteriza y sus representaciones 
geopolíticas cotidianas, así como también su capilarización hacia las políticas nacionales. Es decir, por medio de esta investigación, intentamos avanzar en la reflexión geográfica en el campo de los estudios de frontera patagónica, interrogando las experiencias de un cotidiano que se dinamiza gracias al turismo transfronterizo entre las ciudades de El Chaltén en la provincia de Santa Cruz (Argentina) y Villa O'Higgins, en la región de Aysén (Chile), y los efectos que producen en ella las divergencias entre las políticas de integración y las de seguridad nacionales.

\subsection{Experimentar la frontera en la Patagonia, un ejercicio metodológico.}

Hablar de frontera en Patagonia es un tema que algunos evitan, y que para otros no vale la pena poner en cuestión, pues históricamente la movilidad de las personas en el territorio patagónico se ha sobrepuesto a la existencia misma de una frontera. Las distintas dimensiones de la frontera patagónica han sido abordadas desde las humanidades y las ciencias sociales por investigadores locales de manera exhaustiva, sobre todo en la región de Norpatagonia (ver Martinic 2005, Bandieri 2005, Navarro-Floria 2008, Baeza 2009, Nicoletti et al. 2016, Núñez et al. 2012, Núñez et al 2014, Núñez (Dir) 2016, Núñez et al 2017a, Núñez et al 2018). Respecto al área en cuestión, las publicaciones sobre estudios de frontera en la Patagonia austral, específicamente en la región de los campos de hielo y del lago binacional O’Higgins - San Martín, son bastante más escasas, al menos en ciencias sociales.

Estudiar el habitar alrededor de la frontera en una región donde han existido tensiones diplomáticas reiteradas a lo largo de un proceso histórico de chilenización y de argentinización del territorio, es un asunto bastante sensible. El desafío es realizar un trabajo de campo en una zona fronteriza que se ha mantenido margen del proyecto de integración física entre Chile y Argentina. Es decir, donde no existe un paso fronterizo habilitado por aduanas durante todo el año, ni tampoco hay camino adaptado para el paso vehículos. La ausencia de un vehículo particular restringe el radio de los desplazamientos a aquellos posibles de realizar en buses, en los vehículos de las agencias turísticas locales, y por supuesto, a los senderos que se pueden recorrer a pie. Adicionalmente, el trabajo de campo se complejiza al realizar estos recorridos de manera individual en un área periferizada. Complejidad que se ve aumentada, es este caso, principalmente por el origen, el género, y la edad del sujeto investigador, convirtiendo el trabajo de campo en una experiencia y en un ejercicio reflexivo del cual las emociones forman parte importante (ver Nast 1994; Volvey 2000, 2012). Por lo demás, todos estos aspectos influyen de cierta manera en el surgimiento de una alteridad entre los sujetos de estudio y el(la) investigador(a).

El trabajo de campo se realizó en época estival, en dos periodos diferentes entre los años 2016 y 2018 . Se realizaron 35 entrevistas semi-estructuradas, en Villa O'Higgins, en El Chaltén, en los puestos de control fronterizos y en la localidad de Candelario Mancilla. Se seleccionaron personajes claves, representantes institucionales de la región, habitantes y operadores turísticos. A partir de estos intercambios fue posible comprender algunos aspectos de las formas de habitar en esta latitud de la Patagonia, además de revelar los contrastes entre los modelos de territorialización chileno y argentino. Se consideraron también las conversaciones informales compartidas 
con trabajadores temporales de barcazas y buses, y con turistas en su mayoría de origen europeo. Al respecto, considerar los turistas como actores claves en esta investigación fue muy enriquecedor para evidenciar un habitar transfronterizo de tipo más efímero, pero cuya temporalidad no lo hacía menos importante, pues su circulacion tranfronteriza transforma y dinamiza la integración regional en el plano binacional patagónico. También se consideraron los discursos rescatados luego de una participación como observadora en la reunión del Comité de Integración Austral Sur-Sur que tuvo lugar en El Calafate en marzo del 2017. En ella se pudo corroborar el peso del turismo en la toma de decisiones para la inversión del gasto público, aumentando la proyección de rentabilidad. Por último, se entrevistaron otros actores que fueron claves para entender las dinámicas locales de frontera, tales como militares y policías de los destacamentos regionales, y de los puestos de control de los pasos fronterizos Tenencia Dos Lagunas y puesto de gendarmería Rio El Mosco. Cada dimensión de la frontera es vivida y representada por todo este conjunto de actores de manera diferente, construyendo capas de territorialidad que se imbrican en un territorio patagónico único.

\section{Hacia una geopolítica del cotidiano.}

\subsection{Cambios de paradigma y feminismo en la geopolítica.}

Ha sido un largo proceso el de lograr disociar el uso del término geopolítica de la disciplina puramente militar en manos de jefes de guerra. Si bien fue el sueco Rudolf Kjellén quien diera uso por primera vez al término a fines del siglo XIX, fue realmente Friederich Ratzel quien posicionó la geopolítica a la cabeza de la escuela alemana de geografía, con un impacto bastante global (Encel 2009). Publicada en 1879, la obra Politische Geographie explica los fundamentos del Estado y su relación con el suelo, definiéndolo como un espacio vital. Pero sus planteamientos fueron utilizados en las estrategias de la doctrina del tercer Reich, antecedente que marcó un antes y un después en la geopolítica, influyendo en consecuencia a que su teoría sea considerada como una referencia nazista. Posteriormente surgió entre los especialistas de la materia la necesidad de rescatar el concepto de la geopolítica, siendo principalmente las escuelas geográficas como la francesa y la anglosajona las cuales desarrollaron nuevas perspectivas. Pero a pesar de las innovaciones en la perspectiva, la geopolítica continuaba reproduciéndose en manos de hombres de Estado y se mantenía lejos de las aulas académicas, hasta que paulatinamente se fue integrando como subdisciplina geográfica. En palabras de Rosière, "estos enfoques han sido prácticas de poder mucho antes de ser disciplinas científicas"15 (Rosière, 2007:12). En el caso de Chile, y a diferencia de otros países, la geopolítica ha sido fuertemente influenciada por la obra de Ratzel. Durante muchos años se mantuvo instalada exclusivamente en la esfera militar hasta que se funda el Instituto Geopolítico de Chile en 1981, integrando a académicos, además de otros profesionales del derecho y las ciencias sociales (Child 1979; Santis 1998). Como lo expresa en el título de uno de sus libros, el geógrafo Yves Lacoste, a quien se atribuye la refundación de la escuela geopolítica francesa: 'La geografía sirve, en primer lugar, para hacer la guerra' (Lacoste, 2014). Una frase que buscaba generar la polémica necesaria para abrir el

\footnotetext{
Cita en francés traducida al español por la autora.
} 
debate en torno a una disciplina decaída a nivel pedagógico escolar, pero que se transformó en una herramienta estratégica para la adquisición de poder durante y después de la guerra fría. En este contexto, Lacoste abre el espectro del análisis espacial de la geografía y la define fundamentalmente como "un saber estratégico estrechamente ligado a un conjunto de prácticas políticas y militares"16 (Lacoste 2014: 57). La geografía entonces era considerada como una herramienta que podría cambiar el curso de una batalla, o modificar la distribución espacial de una población interviniendo directamente los factores medioambientales. Esta corriente de la geografía de 'Estados mayores' como la califica Lacoste, se convierte en la base de la refundación de la escuela geopolítica francesa. Si bien esta corriente proponía una renovación disciplinar post segunda guerra mundial, para diferenciarse de las bases epistemológicas de aquellas utilizadas por los geógrafos de la Alemania del tercer Reich, ésta continuó reproduciendo una lectura más tradicional sobre los Estados en una escala global en base a la definición de soberanía westphaliana.

Después de la caída del muro de Berlín cayeron también otros muros, sobre todo en la producción de conocimiento geográfico. De la mano de O' Tuathail (1994) se abrió la escala de análisis de la geopolítica desmarcándola de la rigidez de las fronteras de los Estados-Naciones de la post-guerra, la seguridad y la defensa. El énfasis de la geopolítica critica avanza hacia una multiplicidad de escalas, haciendo énfasis en las escales locales, o glocales, interesándose por los estudios de género y también en las relaciones de poder en un contexto postmodernista. En lo que concierne el estudio de

\footnotetext{
Cita en francés traducida al español por la autora.
}

las fronteras, la geopolítica crítica considera también los procesos internos de producción de fronteras, ya sean sociales, políticos, imaginarios o estéticos: "Ella presta particular atención a las prácticas de delimitación de frontera y a las representaciones que caracterizan la vida cotidiana de los estados"17 (O' Tuathail \& Dalby 1998:3-4).

Sobre la base del cambio de la escala de análisis de Estado-Nación hacia la escala local, los aportes tan importantes como los de Rose (1996), Dowler \& Sharp (2001); Anderson \& Smith (2001), Hyndman (2001), Williams \& Massaro (2013), entre otros, han permitido consolidar una línea de pensamiento feminista en la geografía anglosajona, e inspiran a replantear la posición de las(los) investigadoras(es) en materias tradicionalmente masculinas, como es el caso en el que ha estado reposando la geopolítica en Sudamérica. Esta corriente feminista no se reduce a los estudios de género, pues ella recupera una voz en la investigación que difiere de los estándares masculinos en producción de conocimiento, donde la posición del investigador se distancia del sujeto, y donde el sujeto no tiene cuerpo de representación para visibilizar los problemas geopolíticos en cuestión. El objetivo de la geopolítica feminista es justamente dar lugar para visibilizar las personas quienes representan los efectos de las políticas en la vida cotidiana. La escala representa el eje en el cambio de perspectiva, en relación al análisis de la estructura política y en la producción del espacio (Hyndman 2001).

\footnotetext{
Cita en inglés traducida al español por la autora.
} 


\subsection{La frontera en su dimensión socio- espacial.}

Con los estudios de frontera (border studies) se ha complejizado la noción de frontera, sin conformarse con estudiarla solamente en su dimensión política. Ella ya no se define solamente como una línea invisible divisora de naciones y contenedora de soberanías, sino que se cuestiona como proceso que ha evolucionado bastante desde aquella frontera civilizadora planteada por Turner (1893). Hoy en día podemos entender la frontera como una construcción y re-construcción espacial representada en las prácticas cotidianas de los individuos en una zona fronteriza bastante más fluida (Amilhat-Szary 2015, Paasi 2011). Ella se materializa a través de las acciones que toman lugar en ella, a través de un proceso constante de 'fronterización, es decir:

\begin{abstract}
“(...) las fronteras pueden ser ampliamente definidas como categorías de diferencia que generan distinciones socioespaciales entre lugares, individuos y grupos. Además, como parte de este 'giro' constructivista, la noción de fronterización ha emergido como un contexto general para comprender las fronteras como algo continuamente 'en construcción'." (Kolossov \& Scott 2013:3) ${ }^{18}$.
\end{abstract}

Continuando con la definición de Kolosov \& Scott, podemos decir que la frontera cobra dinamismo y se convierte en una producción territorial donde se imbrican múltiples capas de territorialidad, una estructura espacial con diferentes representaciones de poder superpuestas. Ahora bien, considerando la territorialidad como una expresión especializada de poder, Robert Sack proponía durante los años ochenta lo siguiente:

“(...) la territorialidad se definirá como el intento de un individuo o un grupo para afectar, influenciar, o controlar

18 Cita original en inglés traducida al español por la autora. personas, fenómenos, y vínculos, delimitando y reafirmando el control sobre un área geográfica" (Sack 1986:19).

Sin embargo, esta definición de territorialidad se plantea solamente desde el ejercicio de dominación, dejando de lado otros tipos de relaciones y prácticas de las personas en el espacio, y la expresión de múltiples espacios vividos. Por su parte, Claude Raffestin plantea que la territorialidad se inscribe en un contexto de espacio y tiempo donde la acción humana la determina:

"El paradigma de la territorialidad revierte el orden habitual de la geografía porque el punto de partida no es el espacio, sino que los instrumentos y códigos de los actores que han dejado huellas e indicios en el territorio". (Raffestin 1986:94) ${ }^{19}$.

La territorialidad sería entonces un fenómeno más complejo que puedeexplorarseen diferentes dimensiones de las practicas o acciones humanas espacializadas. Esta producción de territorialidad se extiende también a la frontera, la cual se produce en dimensiones espaciales que se van transformando en un tejido de prácticas humanas cotidianas y viceversa. En este sentido hablamos de 'borderitie':

\section{"En analogía con la territorialidad de cualquier espacio (expresando por qué y cómo el espacio 'hace territorio' o se territorializa a sí mismo), borderitie se refiere a lo que 'hace la frontera' en un espacio dado, desde a un punto de vista funcional y simbólico”20. (Amilhat Szary \& Girault 2015:10).}

De esta manera, podemos entender la territorialidad de la frontera, como equivalente de la 'fronteridad' (borderitie) ${ }^{21}$, la cual se

9 Cita en francés traducida al español por la autora.

20 Cita original en inglés traducida al español por la autora. E concepto de 'borderitie' fue traducido como 'fronteridad'.

21 Podemos traducir borderitie como fronteridad, pero no se ha encontrado ninguna otra traducción fidedigna de este concepto en español que sirva de referencia de validación, por lo tanto, continuaremos utilizando el concepto en inglés, es decir, boderities. 
debe distinguir de la dinámica fronterización / territorialización en relación a las escalas de poder. Esta territorialidad se caracteriza por un conjunto de relaciones que se produce entre los individuos con el espacio de manera cotidiana (Di Méo 1999; Aldhuy 2008), y siendo uno de sus componentes la construcción de identidad o pertenencia (Paasi 2002) en un espacio fronterizo. Entonces, la territorialidad podemos encontrarla también en las prácticas y en las formas de habitar la frontera.

\subsection{Construyendo un concepto de habitar a partir de la territorialidad.}

El desafío se presenta al avanzar en la construcción de una noción de frontera donde la territorialidad esté concebida por el espacio vivido, por las formas de habitar el espacio. Según el "Diccionario Geografía del espacio y de las sociedades", el concepto de habitar se legitima científicamente a partir los años '60s a través de la evolución paulatina de las nociones de 'habitar' y la de 'espacio', nutridas con contribuciones de Eric Dardel, Henri Lefebvre, Martin Heidegger, Gaston Bachelard, Pierre Bordieu, Michel de Certeau (Levy \& Lussault 2003). De esta manera el habitar se fue consolidando como concepto en la escuela francesa de geografía (Paquot et al 2007; Herouard 2007; Stock 2003, 2007, 2012) con reflexiones que nos permitirán hacer el puente con la construcción cotidiana de una geopolítica del espacio fronterizo. Según Levy \& Lussaut (2003), el habitar se puede concebir de múltiples formas, pero considerando siempre el espacio, en el cual la acción de habitar transforma profundamente. Ellos definen el habitar del individuo como un fenómeno espacial y no solo experiencial en el sentido temporal del término (Levy \& Lussaut 2003). Se adiciona una nueva dimensión de análisis a esta relación hombreTierra. Esta se traduce en prácticas que expresan formas de habitar espacializadas en la escala del individuo, pero que se ven multiplicadas en las diferentes prácticas en sociedad. Sobre este aspecto Mathis Stock hace una reflexión que enriquece aún más el concepto, pues propone que existen diferentes modos de habitar que no se restringen solamente a las prácticas del cotidiano, sino que más bien a las prácticas del lugar, a los 'lugares practicados' (Stock 2003). Al ampliar la dimensión temporal de las practicas, podemos considerar, por ejemplo, los turistas en una categoría de habitar más recreativo, con un habitante en tránsito, pero a quien la temporalidad no deslegitimaría su habitar. Stock (2012) valida la experiencia turística como un 'modo de habitar' que genera prácticas en un espacio que es multidimensional y no impreso en la superficie. La duración del habitar, considerando prácticas y rutinas cotidianas en el espacio, sería esencial para distinguir entre modos de habitar permanentes y temporales, y en este último se incluiría al turismo:

\section{“(...) el ejemplo de habitar turístico muestra que las prácticas no rutinarias tienen tanta importancia como las prácticas rutinarias. $Y$ es sin duda el desplazamiento, la discontinuidad espacial efectuada al habitar otro lugar, el cual actúa de mediador en esta distanciación"22. (Stock 2012:69).}

Es decir, la temporalidad de la presencia no sería un criterio para discriminar entre habitante y no habitante, sino que el distanciamiento respecto al lugar. En este caso de estudio, nos referimos a un tipo de turista particular, interesados en impregnarse de la experiencia en el lugar, y establecer vínculos con quienes son nativos o quienes habitan ese lugar. Ellos se diferencian del turista de "tour" en la elección de sus

\footnotetext{
Cita traducida al español por la autora.
} 
rutas y en tiempo de permanencia en el lugar. En la zona fronteriza de la Patagonia Austral podemos distinguir esta categoría de turista/ habitante del turista / visitante, quienes realizan viajes de varias semanas o meses y se toman el tiempo para vivir una experiencia más arraigada con el lugar. Entonces, las diferentes practicas del espacio, en la construcción de distintas formas de habitar, podemos inferir narrativas geopolíticas que emergen de la cotidianidad y prácticas de frontera.

\section{Habitar el espacio fronterizo patagónico y la emergencia de geopolíticas del cotidiano.}

Para comprender la relación entre las formas de habitar y la geopolítica del cotidiano en nuestra área de estudio, es necesario hacer una lectura de las distintas capas de territorialidad de esta frontera que la han construido en tanto que espacio social, natural y político imbricados constantemente. En cuanto a la territorialidad (o fronteridad) en la frontera patagónica, es fundamental considerar que el proceso de definición del límite entre Chile y Argentina tuvo una gran influencia en el posterior ordenamiento territorial de la región, afectando también las posibilidades de desarrollo según la localización de las ciudades y pueblos en relación a la frontera (Sarobe 1943; Nouzeilles 1999; Grenier 1988; Bandieri 2005). Como parte del proceso de colonización y ocupación de los Estados chileno y argentino en la Patagonia, la territorialización de las zonas fronterizas consistió, en gran parte, en la fundación de ciudades, a la presencia de servicios del Estado, y en el levantamiento de monumentos patrióticos con la infaltable presencia de banderas como símbolos de ciudadanía (Baeza 2009). Este proceso de territorialización de la frontera ha sido definido como fronterización, cuyo procedimiento permitía plasmar las soberanías a través de estrategias de nacionalización top-down sobre áreas fronterizas permeables, buscando subordinar hacia un imaginario unitario de pueblo (Nouzeilles 1999; Baeza 2009; Nuñez et al 2014). Otra estrategia en la territorialización de las fronteras fue la creación de parques nacionales. Esta herramienta permitía consolidar los reclamos territoriales de soberanía sobre las grandes extensiones de áreas naturales, controlar los 'espacios vacíos' visibles en los mapas, destinándolas a la conservación y transformándolos en espacios protegidos (Navarro 2008, Núñez et al 2012; Guyot 2011, 2015), convirtiéndose a la vez en 'frentes ecológicos geopolíticos' (Guyot 2017).

Una de las principales particularidades de la frontera en la región patagónica es la permeabilidad del cordón cordillerano (Grenier 1988). En esta latitud de los Andes la frontera naturalquesematerializaenlabarreramontañosa, es interrumpida por la apertura de valles y de grandes lagos, con los cuales favorecen la emergencia de espacios sociales de circulación transfronteriza, constituyendo verdaderos corredores naturales y espontáneos. Como nos recuerda Lefebvre (2000), los espacios sociales no son inertes, se superponen, compenetran, $y$, por lo tanto, no se aíslan en contornos cerrados o límites. Del mismo modo, "las fronteras visibles generan una separación aparente entre los espacios en ambigüedad y en continuidad al mismo tiempo"23 (Lefebvre 2000:104). Pero en la Patagonia, a pesar de la fluidez natural que ofrecen estos espacios, ambos Estados se esfuerzan por imponer sus soberanías las cuales necesitan ser contenidas por márgenes

\footnotetext{
Cita traducida al español por la autora.
} 
estáticos, generando tensiones geopolíticas, y en consecuencia, provocando que ciertas áreas se conviertan en zonas sensibles como es el caso de la Laguna del Desierto y del monte Fitz Roy en las cercanías de los CHS.

Los CHS es una gran región binacional de glaciares que se extiende por sobre unos 16.800 $\mathrm{km}^{2}$, divididos por una frontera difícil de acceder y de controlar. Al mismo tiempo, ellos representan una frontera natural bien evidente, pues se manifiestan como un verdadero obstáculo para la conexión terrestre entre las regiones de Aysén y Magallanes en Chile. Del lado argentino el escenario territorial no presenta el mismo nivel de discontinuidad, sin interrumpir entonces la conectividad de los centros poblados. En la actualidad, los CHS cobran gran importancia en materia de interés geopolítico, pues representan una de las mayores reservas de agua dulce a nivel planetario, mientras que la demarcación del límite internacional sobre su vértice en el monte Fitz Roy permanece pendiente (Manzano 2015). Esta situación genera un velo de incertidumbre al respecto, motivo por el cual las políticas de seguridad y defensa territorial mantienen esta área como inaccesible de manera tácita. Del mismo modo, dicha situación impacta en la configuración territorial de la zona fronteriza tanto en Chile como en Argentina, apresurando la territorialización de la frontera y la afirmación de la soberanía por medio de la creación de parques nacionales y ciudades, procesos que mencionamos anteriormente.

Las ciudades fronterizas más cercanas a estos hitos de tensión diplomática son Villa O'Higgins por el flanco chileno, y El Chaltén por el flanco argentino, rodeadas de una dispersión de estancias y campos los cuales se aproximan aún más al límite internacional. Aunque los procesos de ocupación y colonización del territorio han sido diferentes en esta latitud de la Patagonia (Madsen 1977, Halvorsen 2000, Martinic 2005), no han dejado de estar entretejidos por la experiencia cotidiana de los habitantes de esta zona de glaciares. En ellas, la producción del espacio social contiene también la construcción de formas de habitar (Stock2003), representadas en prácticas de frontera que se ven afectadas por las decisiones de orden geopolítico de un periodo conflictivo entre ambos países. Las políticas nacionales materializaban la "obsesión fronteriza" mencionada por Foucher (2018) por medio de prácticas de seguridad acompañadas por la militarización de la zona luego del tenso episodio de la Laguna de Desierto en 1965, hasta la resolución de una solución pacífica aportada por el laudo arbitral de 1994. Si bien, hoy por hoy las fuerzas armadas de ambos países se han retirado y visitan la zona solo para realizar ejercicios, su presencia marca la experiencia cotidiana de los habitantes y, por consiguiente, en la construcción de la memoria local.

\section{El Parque Nacional Bernardo O'Higgins} (PNBO) fue creado por el Decreto Supremo n. 264 de 1969 en la región de Magallanes, y posteriormente en 1989, a través del Decreto Supremo n. 392, se amplió su superficie a más de 3.500.000 hectáreas entre el océano Pacifico y la frontera con Argentina ${ }^{24}$, de las cuales la mayor parte corresponden a la sección chilena de los CHS. La zona norte de este parque forma parte de la región de Aysén, colindando con los terrenos de los alrededores de Villa O'Higgins. Esta ciudad es el resultado de una antigua ocupación de colonos la cual fue definida como poblado en 1966, y consolidada como comuna

Decretos consultados en la Biblioteca Digital del Congreso Nacional de Chile (www.leychile.cl) el 15/03/2017. 
en 1979 con la ley n. 2.868. La primera decena de familias que llegó a poblar esta zona durante los años 1920 y 1930, ocupó en primera instancia el área montañosa alrededor del rio Mayer. Según las fuentes locales, los ocupantes bajaban a pasar el invierno en el valle, y poco a poco sus viviendas se fueron convirtiendo en su hogar permanente dando origen al pueblo. Si bien el proceso de ocupación y poblamiento de este territorio fue espontaneo, la transformación de este asentamiento en comuna un año después del conflicto de la Laguna del Desierto (1965), corresponde a una estrategia de territorialización por parte del Estado chileno, una "afirmación de chilenidad en zona de frontera" (Martinic 2005:471).

La 'Villa', como le llaman los locales, cuenta con una población estimada de 463 habitantes ${ }^{25}$, entre los cuales algunos son descendientes de los primeros colonos, los ocupantes pioneros. Otros venían constantemente a esta zona desde Cochrane a comprar ganado, y se fueron quedando. También hay aquellos que decidieron instalarse luego de trabajar para el Cuerpo Militar del Trabajo en la construcción de la Carretera Austral. Otros llegaron buscando oportunidades de negocio con la efervescencia turística, y otros respondieron a ofertas laborales del gobierno para trabajar como funcionarios públicos en la escuela local o en la municipalidad ${ }^{26}$. Estas personas se convirtieron en habitantes, y construyen diariamente su relación con el lugar, generan prácticas sociales, comerciales, políticas y de circulación, produciendo un 'vecinaje' regional (voisinage) con Argentina.

Según datos Censo 2002, proyectados al 2006. Consultado en: http://www.subdere.gov.cl/

26 Notas de trabajo de campo, 2017-2018.
Imagen 2. Paso Rio Mayer. El camino se acaba en el cerco que lo separa del territorio argentino.

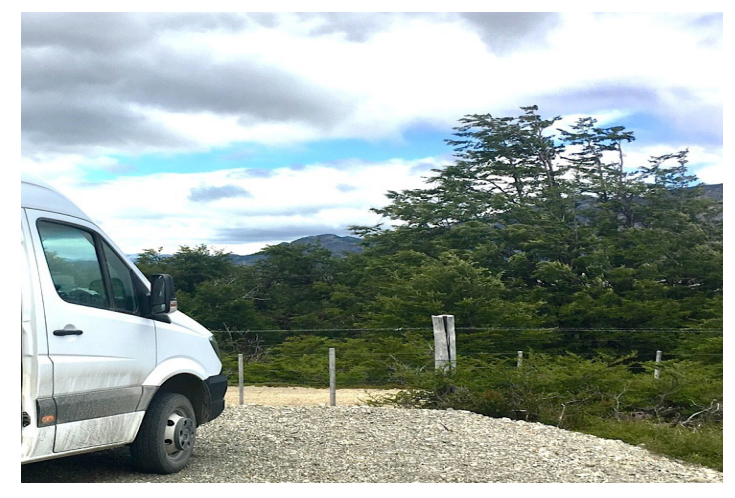

Fuente: Fotografía de la autora.

Como bien lo explica Susana Bandieri respecto a los intentos de territorialización sobre las áreas andinas de la Patagonia, éstas conforman subespacios al margen de un sistema político nacional, donde se generan vínculos comerciales y un espacio social integrado donde funcionaba una fórmula de "cordillera libre" (Bandieri 2005:217). En este contexto, la circulación de chilenos hacia Argentina y el paso de mercaderías hacia Chile se desarrollaba de manera espontánea frente a la condición de aislamiento físico de Villa O'Higgins hasta fines de los años ' 90 . Cruzar la frontera para trabajar durante la temporada estival y volver para pasar el invierno en Chile se convirtió en una práctica del cotidiano patagón:

\footnotetext{
"Yo trabajé muchos años en Argentina, en las estancias. Era la única manera en que la podía sustentar con los artículos de primera necesidad porque acá era súper difícil. Después desde la estancia traíamos todo en pilchero con el caballo. Antes no había control, luego siempre estaba carabineros. Después empezaron a pasar un documento que se llama salvoconduto por si uno se encontraba con la policía argentina y uno pueda justificar que no andabas arrancando ni nada. Era un comprobante que carabineros entregaba al chileno que salía para allá. Antes cuando
} 
agarrábamos la cordillera no sabíamos si estábamos en territorio chileno o en territorio argentino, porque había que por casualidad encontrarse con hito para saber donde es cada país". (Habitante de la zona del rio Mayer 2017).

Imagen 3. Hito Rio Mosco a unos metros de Villa O’higgins.

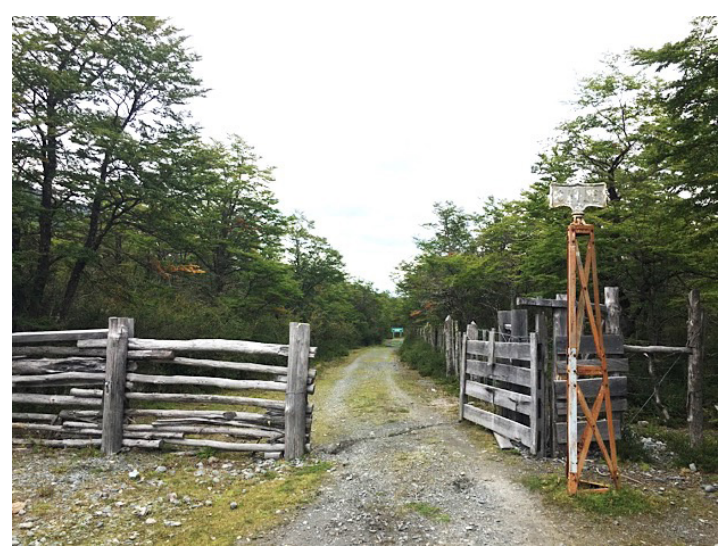

Fuente: Fotografía de la autora.

Los habitantes/ocupantes debieron adaptarse a la presencia de un borde, un límite, de una frontera controlada, a una línea invisible o cerco visible en el paisaje, y marcada con hitos simbólicos de hierro. En este contexto, las circulaciones transfronterizas son también prácticas que definen un régimen de habitar el espacio (Stock 2003), y en el caso de Villa O'Higgins, han ido adaptándose en relación de la mayor presencia del Estado en una zona marcada por el aislamiento geográfico. A pesar de estas restricciones, la circulación, el ir y volver, se mantiene en el tiempo entre quienes continúan con la crianza de ganado, pero a una escala mucho menor.

Para quienes han construido una forma de habitar asumiendo las condiciones del lugar, el aislamiento es un factor relativo y no se sobrepone al desarrollo de un sentimiento de arraigo con la tierra (Tuan 1974). La distancia con los centros de poder hacia el norte y la discontinuidad territorial hacia el sur, si bien marcan una condición de marginalidad no se traducen en aislamiento (Núñez et al. 2017b). Adicionalmente, el calentamiento global y el aumento de las temperaturas locales han modificado el paisaje al disminuir la cantidad de nieve caída anualmente, influyendo así en la periodicidad de los desplazamientos:

"Si estaba bueno, en noviembre se podía llevar animales
a Cochrane. Y antes de noviembre no se podía, luego
llegaba marzo y cerraban la cruzada por la nieve. Había
un avión que llevaba animales a Cochrane, pero si nevaba
mucho pasaban 15 días y no había avión. Ahí estábamos
aislados. Ahora tenemos avión dos veces a la semana.
Antes estábamos aislados, porque nevaba mucho".
(Habitante descendiente de colonos pioneros, villa
O'Higgins 2017).

Habiendo estado esta zona de la Patagonia dedicada principalmente a la producción agrícola-ganadera, la llegada de las instituciones del Estado como es el caso de la Corporación Nacional forestal (CONAF) obligó a cambiar las prácticas en el monte, lo que conllevó a una disminución importante de la ganadería local. Su impacto generó una transformación de las formas de habitar, de las prácticas cotidianas, de la relación hombre-naturaleza. Era una época donde se vivía de "hacer campo", como cuenta, uno de los habitantes nacidos en el lugar. Se despejaba, se talaban y se quemaban árboles para crear praderas, y de esta manera evitar la "maleza". Esta práctica cambió con la llegada de las instituciones del Estado:

"La llegada del agro no fue nada buena para nosotros, CONAF, INDAP, el SAG. Porque esto son todos campos en cordillera virgen, cordillera natural, con maleza natural. Entonces antes de que el agro llegara, la gente rozaba sus campos, lo quemaba. Rozar es limpiar, cortar toda 
la maleza ya sea coihue o canelo, lo que sea, para poder sembrar pasto. Pero CONAF nos dijo que estábamos infringiendo la ley, que podíamos ir a la cárcel. Entonces la gente no pudo hacer más campo. Esto ahora lo ves lleno de maleza, eso para la parte turística es impecable, por los paisajes espectaculares, pero para la gente campesina no. Las vacas no comen maleza. Entonces no podíamos hacer nada. Teníamos 2.900 hectáreas, ¿De qué sirve estar aquí si no podemos tener animales? Todos vendimos". (Habitante oriundo de la zona del Rio Mayer).

La quema de bosques para convertirlos en campos cultivables se convirtió en una práctica común durante el periodo de colonización de la región de Aysén (Martinic 2005). Hacer campo era requisito para ser colono en Aysén desde principios del siglo $\mathrm{XX}$, conforme a las exigencias del DFL 256 del Ministerio de Tierras y Colonización. La abundancia de bosques y la necesidad de espacio pastoril productivo justificaban la implementación de la técnica de quema como efecto del "discurso del desarrollo" del proceso de colonización del Estado (Núñez et al. 2014), sin preocuparse por los efectos futuros de la antropización de la naturaleza. Según los relatos de los habitantes entrevistados, el uso de la quema iba acompañado de la creación de un cordón cortafuego alrededor del área. Este complemento de técnicas se puede interpretar como una construcción local de conocimientos que no contraponen al hombre y la naturaleza en un intento de dominación, sino más bien de 'vivir con', conformando saberes 'tecnoambientales" (Ingold 2000:320).

En las costas chilenas del Lago O'Higgins / San Martín, junto con la familia Mancilla, otras diez se instalaron al borde del lago (Martinic 2005). Sus predios no cuentan con conectividad terrestre con Villa O'Higgins hacia el norte, ni hacia Argentina, y se encuentran rodeados hacia el sur por el Parque Nacional Bernardo O’Higgins y los CHS. Por lo mismo, hasta el día de hoy, algunos de ellos siguen trabajando como temporeros en las estancias argentinas, y son completamente dependientes de la conexión lacustre quincenal que ofrece una barcaza subvencionada por el gobierno para transportar animales y mercaderías. Las dificultades de acceso a la localidad de Candelario Mancilla la posicionan como la más aislada de la región de Aysén, y una de las más aisladas del país, según propuesta de la Política Regional de Localidades Aisladas. Este instrumento define a su vez la política de territorialización:

“(...) a través de políticas especiales se pueden apoyar estos asentamientos humanos y estimular el desarrollo de zonas especiales en el contexto de soberanía, ya que en el caso de la zona en litigio es el último lugar del territorio sin explorar"27.

Esta política de tono geopolítico es el resultado de la evolución de antiguas directrices de planificación del espacio nacional donde se definían los territorios prioritarios para la acción del Estado. En los primeros diseños de políticas de ordenamiento territorial creadas por organismos estatales como la CORFO, la ODEPLAN y la CONARA, durante las décadas '60s y '70s, se definieron conceptos tales como como 'polos de desarrollo', 'zonas de colonización', o 'zonas extremas'. Pese a interesarse en las zonas periféricas, en estas políticas se consideraban escalas de macrozonas regionales o provinciales, sin enfatizar la necesidad de acercar el Estado a los centros poblados fronterizos. Posteriormente, el concepto de 'frontera interior' desarrollada por el Ejército de Chile durante los años '70, abrió la discusión sobre la necesidad de incluir las comunidades fronterizas y aisladas a la dinámica nacional, asumiendo el margen del país como

SUBDERE Aysén. 2012. Propuesta regional de localidades aisladas, p. 25. 
periferia (Rodríguez et al. 2018). Podemos inferir entonces que los esfuerzos de territorialización a través de los instrumentos de ordenamiento territorial son una estrategia para imponer la soberanía en espacios de débilmente poblados y también en los no poblados (Foucault 2009).

Imagen 4. Esquema de la ruta del cruce fronterizo entre El chaltén y Villa O'Higgins

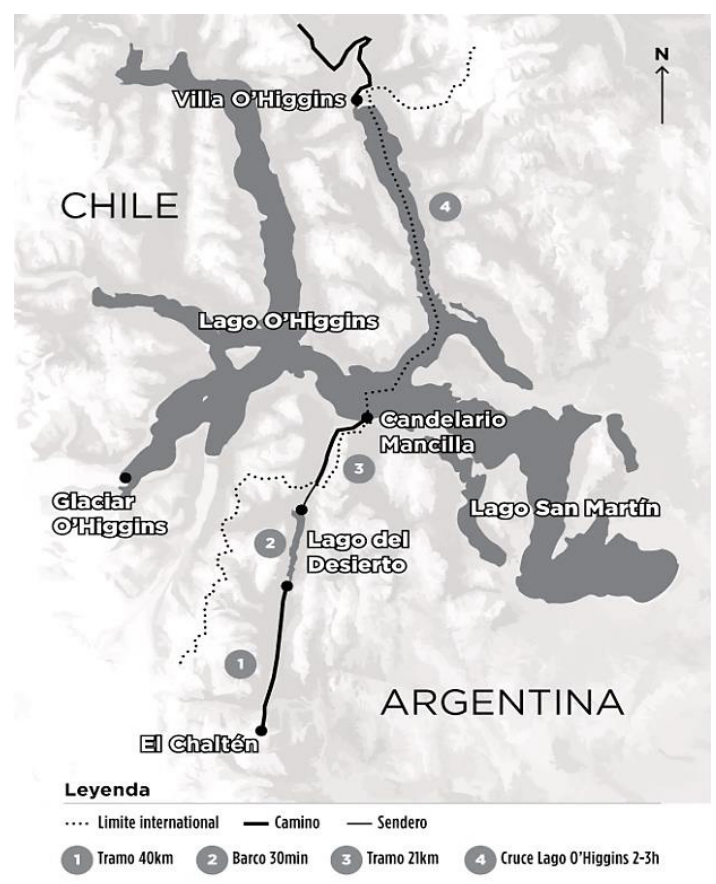

Fuente: Creado por la autora en base a mapas turisticos.

En la actualidad, cerca de esta localidad se instaló el paso fronterizo "Dos Lagunas" donde existe una presencia constante de la Escuela de Frontera de carabineros quienes mantienen abierto el paso fronterizo entre los meses de octubre y abril. Desde aquí el retén Teniente Merino controla el flujo de migraciones de la frontera por donde circulan cerca de 3.000 personas por año. La movilidad que caracteriza este lugar corresponde principalmente a turistas nacionales y extranjeros que realizan circuitos de turismo transfronterizo, especialmente de origen francés, alemán y suizo ${ }^{28}$. La ruta entre El Chaltén y Villa O'Higgins es parte de un circuito binacional en el cual la localidad de Candelario Mancilla es una parada obligada. El flujo de turistas por este paso fronterizo hacia Villa O'Higgins, además de aquellos que llegan dese el norte a través de la carretera austral, sustentan y dinamizan la economía local, transformándola cada vez más dependiente del turismo.

Parafraseando a Stock, en la Patagonia, el turista también "haría con el espacio", desarrollaría prácticas que producen habitar. En este sentido, el turista también transformaría el espacio patagónico contribuyendo a acelerar la integración regional entre Chile y Argentina (Lagesite 2017), conformando un frente turístico transfronterizo con circuitos que ayudan a cambiar el carácter periférico o marginal atribuido a la Patagonia como "fin del mundo". A diferencia del entusiasmo de los turistas por los paisajes que ofrecen los glaciares de los CHS, ellos no son percibidos de la misma manera por los habitantes locales:

\footnotetext{
"Yo he ido a trabajar en los campos por allá cerca de la central, he ido, he visto el ventisquero. Pero que a mi me haya llamado la atención para decir 'voy a ir a ver ese ventisquero, ese hielo', no iría. Porque si me críe con la nieve hasta el cuello, estuve rodeado de nieve desde chico, y ¿ahora de grande voy a ir a ver el hielo? Antes nevaba mucho, ha cambiado mucho". (Habitante, Candelario Mancilla 2017).
}

Podemos inferir que la apreciación de la naturaleza y del wilderness como paisaje con

\footnotetext{
Cifras otorgadas por carabineros.
} 
atributos de belleza escénica, podría depender del tipo de espectador visitante o nativo y de su experiencia de vida (Tuan 1974).

En Argentina, el área equivalente al caso de Villa O’Higgins, es la ciudad de El Chaltén, la cual se encuentra en un área desafectada al interior del Parque Nacional Los Glaciares (PNLG) en la Provincia de Santa Cruz. Este parque fue definido en primer lugar primero como área protegida por medio del decreto n.105.433 en 1937, y consolidado como parque nacional en 1971 con la ley n. 19.292 con una superficie de 726.927 hectáreas $^{29}$. En la anexión de nuevos terrenos bajo la protección del parque Los Glaciares durante los años '70s, se incluye la Reserva Nacional de la zona norte Viedma, donde se encuentra el monte Fitz Roy y también la ciudad de El Chaltén. El decreto de esta zona como parte del parque habría sido posterior al conflicto de la Laguna del Desierto (Halvorsen 2000), para asegurar la protección del territorio de manera estratégica en zona de frontera de seguridad. Años más tarde, en 1985, se decretó a El Chaltén con la categoría de 'población' por medio de la ley n. $1771^{30}$. Luego en 1990, a través de la ley n. 23.766, se desafectaron 135 hectáreas para dar paso al tejido urbano.

El poblamiento de El Chaltén corresponde a un proceso menos espontaneo que la de su vecina Villa O'Higgins. Se trata más bien de una estrategia de ocupación territorial que permitía fortalecer la soberanía argentina en la zona del conflicto, pues en un principio esta área fue ocupada por un campamento militar durante

29 Decretos consultados en la plataforma de Información Legislativa y Documental del Ministerio de Justicia y Derechos Humanos de Argentina (http://www.infoleg.gob.ar). 20/05/2017.

30 Consultado el 12/05/2017 en el Dirección Nacional del Sistema Argentino de Información Jurídica (SAIJ): (http://www.saij.gob.ar) el periodo de tensiones limítrofes con Chile por la Laguna del Desierto en los años 1960. Este campo se transformó paulatinamente en un poblado con las familias de los militares, siendo estas primeras casas construidas por el Estado.

Imagen 5. Vista del monte Ftiz Roy desde la entrada de El Chaltén.

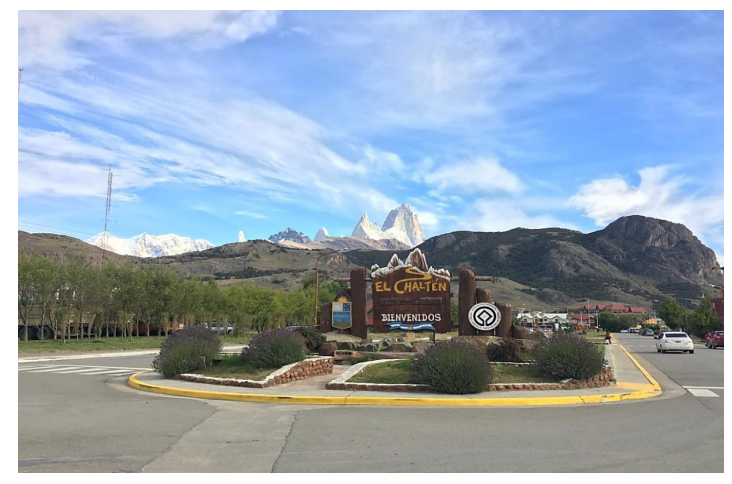

Fuente: Fotografía de la autora.

El Chaltén es uno de los pueblos más jóvenes de Argentina, poblado por unos 1600 habitantes quienes han debido acomodarse a las limitaciones de los terrenos estatales disponibles para ser adjudicados sin sobrepasar los límites del tejido urbano, lo cual ha ido generando actualmente una verdadera crisis habitacional y de la propiedad de la tierra. Una buena parte de los habitantes fijos de El Chaltén arrienda vivienda, a la espera de obtener una adjudicación de los pocos terrenos que se van sorteando periódicamente.

\footnotetext{
“Acá lo complicado para vivir en El Chaltén es la vivienda y el trabajo todo el año. La vivienda es lo más complicado. No crece en superficie, son 130 hectáreas el pueblo, y que fue la primera y única desafectación que hizo parques nacionales para fundar el pueblo, y listo". (Habitante, El Chaltén 2018)
} 
Aun así, la cantidad de habitantes puede duplicarse $o$ incluso triplicarse durante la temporada de verano y la llegada de los trabajadores "golondrinas", generándose un sistema de arriendo de viviendas donde la demanda ha elevado los precios significativamente. Entonces, el habitar en esta ciudad también está marcado por la temporalidad de las estaciones del año. La temporada se inaugura el 12 de octubre, fecha definida por ley como día fundacional de la ciudad, y se clausura en marzo o abril con la celebración de semana santa. Esta periodicidad marca el ritmo del habitar del Chaltén, e incluso el calendario escolar se adapta a este periodo. Durante el invierno no hay clases escolares debido a las dificultades de traslado a causa de la nieve, y más de la mitad de los habitantes se van del pueblo para volver a trabajar en la próxima temporada:

\footnotetext{
"Golondrinas les llamamos. Lo que es la vida del pueblo, de los residentes permanentes, está un poco diluida, porque es más la gente que viene a trabajar que la realmente son residentes. Directa o indirectamente, todos dependemos del turismo. Finalmente, el pueblo es lo que es por el turismo". (Habitante, El Chaltén 2018).
}

La consolidación política de El Chaltén ha sido un proceso lento desde la formación de la Sociedad de Fomento hasta las primeras elecciones de intendente, las cuales tuvieron lugar en el 2015. Su primer intendente electo, Raúl Andrade, es descendiente de pioneros chilenos que poblaron el sector del Lago del Desierto:

"Luego del conflicto por la Laguna del Desierto en 1965, mis tíos que eran argentinos se fueron a vivir a Villa O'Higgins. (...) Pero para mí no hay frontera. Ir a Villa O'Higgins o ir a Chile Chico para mi es lo mismo. Es un valle que nos divide con un río, pero para mí es lo mismo. Nosotros tenemos familia chilena y también argentina. Yo no veo un límite, o que haya una distancia entre Chile y Argentina, para mi es todo lo mismo". (Intendente, El Chaltén 2017).
La masificación del turismo favorecida por la pavimentación del camino que los conecta con El Calafate, ha ayudado a poner el pueblo sobre el mapa y conseguir recientemente la instalación de servicios básicos. Hoy en día el contraste es visible en relación a el desarrollo del turismo en el lado chileno. Durante la temporada 20172018, la sección norte del Parque Nacional Los Glaciares recibió un total 150.000 visitantes los cuales ingresan desde El Chaltén ${ }^{31}$. En este parque el monte Fitz Roy se ha transformado en el principal atractivo turístico de los amantes de la escalada, coronándose como un haut lieux del turismo patagónico. Cada año son mayores los flujos de turistas que cruzan la frontera desde el PNLG hacia los CHS guiados por especialistas, ya sea a través del Paso Marconi o el Paso de Los Vientos, ambos sectores que no cuentan con un puesto de control aduanero fijo. A pesar de las dificultades en la administración del parque, la condición fronteriza que lo hermana con el Parque Nacional Bernardo O'Higgins, no existe una plataforma integrada para la protección de las áreas protegidas fronterizas:

\footnotetext{
"No tiene sentido que discutamos sobre el hielo, y que no lo conservemos entre los dos países. Nos deberíamos juntar periódicamente CONAF con Parques Nacionales y trabajar en conjunto, no superponer esfuerzos de estudios, no superponer esfuerzos de gente, de control, de todo. No tiene sentido que nosotros organicemos una recorrida nuestra gastando un montón de gente, mientras que al mismo tiempo está realizándolo CONAF para fiscalizar lo mismo". (Funcionario Parques Nacionales, El Chaltén, 2017).
}

En cuanto a las aspiraciones de conectividad entre Villa O'Higgins y El Chaltén, se han abierto discusiones al respecto, pero no todos están de acuerdo con abrir un camino para conectarse con la localidad de Candelario Mancilla, pues significaría promover el turismo masivo de

\footnotetext{
Notas de trabajo de campo.
} 
en una reserva natural donde se encuentra la Laguna del Desierto:

\begin{abstract}
"Ahí está el límite norte del parque, entonces no hay otra población, para allá como que no hay nada para nosotros. Por este lado del Lago del Desierto no estamos realmente conectados con Villa O'Higgins. De todas maneras, es una movida más comercial, no impacta en la sociedad de acá, o por lo menos de como yo la vivo, esta conexión con Villa O'Higgins. Villa O'Higgins es muy muy remoto para nosotros. Yo lo veo re lejos Villa O'Higgins. Chile en general para nosotros está lejos, por más que estemos re cerquita, no lo tenemos presente. Si tenemos presente el motivo por el cual se fundó el pueblo, eso todos lo sabemos, pero no hay, no está presente la frontera". (Habitante, El Chaltén, 2018).
\end{abstract}

De manera alternativa, se ha presentado un proyecto para implementar la infraestructura necesaria para conectar ambas comunidades a través del Paso Rio Mayer, para lo cual faltan solo asfaltar un camino de $90 \mathrm{~km}$ y construir un puente de 50 metros en el lado argentino. Pero esta discusión se ha prolongado por años desde las reuniones binacionales de las autoridades locales, las reuniones anuales del Comité de Integración Austral Sur-Sur, y las reuniones de Cancillería, pero en ninguno de los espacios se ha logrado avanzar con un compromiso de verdadera integración territorial.

\section{Reflexiones finales}

A través de esta investigación hemos podido confirmar que las geopolíticas emergentes desde las dinámicas del habitar cotidianas, expresadas en las narrativas y discursos de los entrevistados, corresponden a procesos en constante transformación en cuanto a la relación del habitante y el espacio fronterizo, construyendo en el tiempo una amalgama de capas de territorialidad que sobrepasa los límites de un Estado-Nación. Las tensiones geopolíticas de los episodios de conflicto diplomático y militar, si bien promovieron el separatismo entre las comunidades chilenas y argentinas reforzando las prácticas deseguridad fronteriza y los discursos nacionalistas, no fueron suficientes para transformar un habitus de circulación e intercambio comercial entre ambos países. En este sentido, las prácticas de circulación transfronterizas en tanto que formas de habitar se han ido renovando en relación a las transformaciones económicas nacionales y al posicionamiento del turismo como actividad económica creciente en la región. En la actualidad no son los habitantes locales los que circulan por esta frontera como en antaño, sino que los habitantesturistas quienes reproducen estas prácticas transitorias. El uso actual del espacio fronterizo y el interés por desarrollar del turismo de manera integrada entre ambas comunidades, visibiliza una contracorriente geopolítica desde lo local y manifestada en la cotidianeidad, sobre todo desde la comunidad de Villa O'Higgins, cuyos habitantes son los más afectados por la marginalización y las restricciones de su espacio fronterizo como efecto de las tensiones del pasado y a su aislamiento relativo. En cuanto a las aspiraciones de conectividad rutera entre ambas ciudades, la lentitud de las negociaciones del Comité de Integración Austral, ha impulsado a las autoridades locales y a las cámaras de comercio a generar sus propias plataformas de discusión, surgiendo en ellas una para-diplomacia espontánea con el objetivo de discutir sobre el modelo de turismo que conllevaría un tránsito masivo en las cercanías de los CHS. Esta iniciativa bottom-up nos confirma que una geopolítica del cotidiano de escala local intenta articularse en el plano binacional. Pero si bien, para los habitantes de Villa O'Higgins es un tema 
urgente romper con su condición de culde-sac ${ }^{32}$, para los de El Chaltén no es un proyecto que trasforme ni su economía ni su accesibilidad. Paralelamente, el silencio de las autoridades nacionales al respecto revela la insistencia por aislar esta frontera de glaciares, generando una contradicción entre los esfuerzos por mantener una política de seguridad de zonas fronterizas en paralelo a los esfuerzos de integración.

\section{Bibliografía}

Aldhuy, A. 2008. Au-delà du territoire, la territorialité ?. Géodoc, pp.35-42.

Amilhat-Szary, A. 2005. Géopolitique et frontières en Amérique Latine. En Hardy, S. ; Médina, L. L'Amérique Latine, Editions du Temps, pp.11-33, Questions de Géographie.

2015. Qu'est-ce qu'une frontière aujourd'hui ?. Presses Universitaires de France, Paris.

Amilhay Szary, A.; Giraut, F. (Eds.). 2015. Borderities: The Politics of Contemporary Mobile Borders. Palgrave Macmillan.

Anderson, K.; Smith, S. 2001. Emotional Geographies. Transaction of the Institute of British Geographers, vol. 26, n. 1: 7-10.

Baeza, B. 2009. Fronteras e identidades en Patagonia central (1885-2007). Rosario, Pro-historia ediciones.

Bandieri, S. 2005. Historia de la Patagonia. Ed. Sudamericana. Child, J. 1979. Geopolitical Thinking in Latin America. Latin American Research Review, vol. 14, n. 2: 89-111.

Di Méo, G. 1999. Géographies tranquilles du quotidien. Une analyse de la contribution des sciences sociales et de la géographie à l'étude des pratiques spatiales. Cahiers de géographie de Québec, vol 43, n. 118.

Dowler, L.; Sharp, J. 2001. A feminist geopolitics? Space \& Polity, vol. 5, n. 3 : 165-176. Paris.

Encel, F. 2009. Comprendre la géopolitique. Editions du Seuil,

Foucault, M. 2009 (2004). Seguridad, Territorio, Población. Curso en el Collège de France (1977-1978). Fondo de Cultura Económica.

Foucher, M. 1991 [1988], Fronts et frontières. Un tour géopolitique. Fayard.

2018. Frontières : rejet, attachement, obsession. Revista Pouvoirs, vol. 2 n. 165 : 5-14.

García Jiménez, M. 1996. La Sentencia Arbitral de 21 de octubre de 1994: "Controversia sobre el recorrido de la traza del límite entre el Hito 62 y el Monte Fitz-Roy" (Laguna del Desierto) (Chile/Argentina). Anuario español de derecho internacional, n. 12: 279-405.

32 Expresion francesa que hace alusión a una zona o camino $\sin$ salida.
Grenier, P. 1988. "L'effet-frontière » dans l'utilisation et l'organisation de l'espace des Andes argentines. Revue de Géographie Alpine, vol. 76 n 1: 7-44.

Guyot, S. 2011. The eco-frontier paradigm: rethinking the links between space, nature and politics. Geopolitics, Taylor \& Francis (Routledge), 16 (3), pp. 675-706.

2015. "The politics of eco-frontiers: when environmentality meets borderities". En Amilhat-Szary \& Giraut (Eds). Borderities: the politics of post-modern borders, Palgrave Macmillan

2017. La nature, l'autre frontière. Fronts écologiques au Sud (Afrique du Sud, Argentine, Chili). Peter Lang ed., Bruxelles

Halvorsen, P. 2000. Between the Rio de las vueltas and the Continental Ice Cap. Editociones El Calafate.

Herouard, F. 2007. "Habiter et espace vécu : une approche transversale pour une géographie de l'habiter ». Habiter, le propre de l'humain. Villes, territoires et philosophie. Paquot, T. et al. La Découverte. 159-170.

Hyndman, J. 2001. Towards a feminist geopolitics. Canadian Geographer / Le Géographe canadien 45(2) : 210 - 222.

Ingold, T. 2000. The perception of the environment. Essays in livelihood, dwelling and skill. Routledge, London.

Kolossov, V.; Scott, J. 2013. Selected conceptual issues in border studies. Belgeo, Revue belge de géographie, n. 1. URL : http://belgeo.revues.org/10532

Lagesite, J. 2017. «Bout de monde en Patagonie ». Tourisme et périphéries. La centralité des lieux en question. En Bernard, N., Blondy, C., Duhamel, P (Eds.). Presses Universitaires de Paris, pp. 181-195.

Lacoste, P. 1999. El papel de la frontera en las relaciones argentino-chilenas. Revista Diplomacia, n. 79: 43-58, abril-junio 1999.

Lacoste, Y. 2014 (1976). La géographie, ça sert d'abord, à faire la guerre. La découverte, Paris.

Lefebvre, H. 2000. La production de l'espace. $4^{e}$ édition. Editorial Anthropos, Paris.

Levy, J. ; Lussault, M. (Dir). 2003. Dictionnaire de la Géographie. Ediciones Belin, Paris.

Madsen, A. 1977 (1975). Patagonia vieja. Editorial Galerna, Buenos Aires.

Manzano, K. 2015.Campos de hielo sur: el agua y su rol 
geopolítico. Revista de Relaciones Internacionales, Estrategia y Seguridad, vol. 10, n. 2:131-151.

Martinic, M. 2005. De la Trapananda al Aysén: una Mirada reflexiva sobre el acontecer de la Región de Aysén desde la prehistoria hasta nuestros días.

Nast, H. 1994. Women in the field: Critical feminist methodologies and theoretical perspectives. The Professional Geographer, vol. 46, issue 1:54-66.

Navarro Floria, P. 2008. El proceso de construcción de la región del Nahuel Huapi en la práctica simbólica y material de Exequiel Bustillo (1934-1944). Revista Pilquen, sección Ciencias Sociales, año IX, N 9.

Nicoletti, M. A.; Núñez, A.; Núñez, P. (Eds). 2016. AraucaníaNorpatagonia. Discursos y representaciones de la materialidad. Editorial UNRN.

Nouzeilles, G. 1999. Patagonia as a Borderland: Nature, Culture and the idea of the State. Journal of Latin American Cultural Studies, vol. 8, n. 1: 35-48.

Núñez, A.; Aliste, E.; Bello, A. 2014. El discurso del desarrollo en Patagonia-Aysén: la conservación y la protección de la naturaleza como dispositivos de una renovada colonización. Chile, siglos XX-XXI. Scripta Nova, Revista electrónica de Geografía y Ciencias Sociales, vol. XVIII, n. 493 (46).

Núñez, A.; Aliste, E.; Bello, A.; Osorio, M. 2017a. Imaginarios geográficos, prácticas y discursos de frontera. Aisén-Patagonia desde el texto de la nación. Editorial LOM, Santiago.

Núñez, A.; Baeza, B.; Benwell, M. 2017b. Cuando la nación queda lejos: fronteras cotidianas en el paso Lago Verde (AysénChile) - Aldea Las Pampas (Chubut-Argentina). Revista de Geografía Norte Grande, 66:97-116.

Núñez, P.; Matossian, B.; Vejsbjerg, L. 2012. Patagonia, de margen exótico a periferia turística. Una mirada sobre un área natural protegida de frontera. PASOS. Revista de Turismo y Patrimonio Cultural, vol. 10, n. 1, enero, pp. 47-59.

Núñez, P (Dir). 2016. Fronteras Conceptuales. Fronteras Patagónicas. Viedma. Editorial UNRN.

Núñez, P., Núñez, A.,Tamagnini, M., Matossian, B. y Odone, C. (dir.) 2018. Araucania-Norpatagonia II. La fluidez, lo disruptivo y el sentido de la frontera. Viedma. Editorial UNRN.

O' Tuathail, G; Toal, G. 1994. Critical Geopolitics and Development Theory: Intensifying the Dialogue. Transactions of the Institute of British Geographers, vol. 19, n. 2, pp. 228- 233.

O' Tuathail, G., Dalby, S. 1998. "Introduction: Rethinking geopolitics. Towards a critical geopolitics". Rethinking Geopolitics. O' Tuathail, G. y Dalby, S. (Eds.). Routledge, London. 1-15.

Paquot, T., Lussault, M., y Younès, C. (dir). 2007. Habiter, le propre de l'humain. Villes, territoires et philosophie. La Découverte.

Paasi, A. 2002. Bounded spaces in the mobile world: deconstructing 'regional identity'. Journal of social and economic geography, vol. 93, n. 2, pp. 137-148.

2011. "A Border Theory: an unattainable dream or a realistic Aim for Border Scholars?". The Ashgate research companion to border studies. Wastl-Walter, D. (Ed.). Ashgate:11-31.

Raffestin, C. 1986. Territorialité : Concept ou Paradigme de la géographie sociale? Revista Geographica Helvetica, n. 2 : 91-96.

Rodríguez, J.; Gissi, N.; Mandujano, F. 2018. Fronteras internas y hegemonías predictivas en Chile: El caso de la Patagonia Austral. Revista Chungará de Antropología Chilena, vol. 50, n. 4: 633-650.

Rose, G. 1996 (1993). Feminism \& Geography. The limits of geographical knowledge. Polity Press.

Rosière, S. 2007. Géographie politique \& Géopolitique. Editions Ellipses.

Sarobe, J. 1943 (1934). La Patagonia y sus problemas. Estudio geográfico, económico, político y social de los territorios nacionales del sur. Editorial Guillermo Kraft, Buenos Aires.

Sack, R. D. 1986. Human territoriality. Its theory and history. Cambridge University Press, London.

Santis, H. 1998. El pensamiento geográfico-político de Ratzel en la geopolítica chilena. Revista de geografía Norte Grande, $\mathrm{n}$. 25, pp. 135-140.

Secor, A. 2001. Towards a feminist Counter-geopolitics : Gender, Space and Islamist Politics in Istambul. Space \& Polity, vol. 5, n.3: 191-211.

Stock, M. 2003. Pratiques des lieux, modes d'habiter, régimes d'habiter: Pour une analyse trialogique des dimensions spatiales des sociétés humaines. Travaux de l'Institut Géographique de Reims, vol. 29-30, n¹15-118, pp. 213-229.

2007. «6. Théorie de l'habiter. Questionnements ». En Paquot, T., Lussault, M., y Younès, C. (dir). Habiter, le propre de l'humain. La Découverte Armillaire, pp. 103-125.

2012. "Faire avec de l'espace: pour une approche de l'habiter par les practiques". En Frelat-Kahn, B. \& Lazzarotti, O. (dir). Habiter. Vers un noveau concept?. Ediciones Armand Colin, Recherches. Paris. Pp. 57-75.

Tuan, Yi-Fu. 1974. Topophilia. A study of environmental perception, altitudes, and values. Prentince-Hall Inc, New Jersey.

Turner, F. 1893. The Significance of the Frontier in American History. Annual Report of the American Historical Association, 199-205.

Volvey, A. 2000. L'espace, vu du corps. En Lévy

J., Lussault M. (dir.), Logiques de l'espace, esprit des lieux. Géographies à Cerisy, Paris, Belin, 319-332.

Volvey, A., Calberac,Y., Houssay-Holzschuch, M. 2012. "Terrains de je. (Du) Sujet (au) géographique ». Annales de Géographie, 687-688 : 5, pp. 441-459.

Williams, J.; Massaro, V. 2013. Feminist Geopolitics: Unpacking (in)security, animating social change. Geopolitics, n. 18, pp. 751-758. 
